EPJ Web of Conferences 41, 02026 (2013)

DOI: 10.1051/epjconf/20134102026

(C) Owned by the authors, published by EDP Sciences, 2013

\title{
Efficient attosecond control of electron dynamics in molecules
}

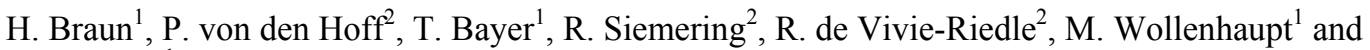 \\ T. Baumert ${ }^{1}$ \\ ${ }^{1}$ Institut für Physik und CINSaT, Universität Kassel, Heinrich-Plett-Str. 40, 34132 Kassel, Germany \\ ${ }^{2}$ Department Chemie, Ludwig-Maximilians-Universität München, Butenandt-Str. 11, \\ 81377 München, Germany
}

\begin{abstract}
We demonstrate how the fast electron dynamics in molecules and hence the reaction of the system can be efficiently manipulated by controlling the temporal phase of an ultrashort laser pulse with attosecond precision.
\end{abstract}

\section{Molecular system and control scheme}

Femtosecond laser pulses interact with matter on the time scale of nuclear motions. Even shorter laser pulses down to the attosecond time regime became experimentally available and raised the hope to measure and control the fast electronic processes. While attosecond laser pulses are an excellent tool to observe ultrafast processes, they may not be the first choice for the control of valence bond chemistry. Due to the high photon energy (XUV) of attosecond laser fields, they address the inner shell electrons. Electronic excitations of the valence electrons that are driven by pico- to femtosecond laserpulses in the visible range on the other hand benefit from large transition moments, which are a requirement for efficient population transfer.

The coherent control scheme presented here utilizes resonant, intense femtosecond laserpulses shaped with attosecond precision [1]. With this scenario the electron motion in a molecule can be controlled to steer the molecule selectively into different electronic target channels - each of which is associated with different subsequent nuclear dynamics. The scheme was discussed theoretically on the potassium dimer $\left(\mathrm{K}_{2}\right)$ serving as a molecular prototype system $[2,3]$. Now we report on the successful experimental realization of this switching mechanism in molecules. In addition we show with the help of quantum dynamical calculations, that control in the coupled electron-nuclear system is achieved by the proposed scenario.

The scheme for excitation of $\mathrm{K}_{2}$ with near $800 \mathrm{~nm}$ laserpulses (photon arrows) is shown in Fig. 1 . When irradiated by a weak laser field, the molecule is excited perturbatively from its groundstate $\mathrm{X}^{1} \Sigma^{+} \mathrm{g}$ via the resonant intermediate state $\mathrm{A}^{1} \Sigma^{+}$u to the target state $2^{1} \Pi_{\mathrm{g}}$. In the strong-field regime however the PESs are modified by the intense laser field and thus the population transfer to the target states is altered. The strong light-induced coupling of the groundstate and the resonant $\mathrm{A}^{1} \Sigma^{+}{ }_{\mathrm{u}}$ state leads to the formation of an electronic wave packet. In a spatiotemporal picture this photoinduced charge oscillation gives rise to an oscillating electric dipole moment $\vec{\mu}(t)$. The phase relation between the driving laser field $\vec{E}(t)$ and the induced dipole moment determines the energy $\varepsilon(t)$ of the interacting system as $\varepsilon(t)=-\vec{\mu}(t) \cdot \vec{E}(t)$ by the relative orientation of $\vec{E}$ and $\vec{\mu}$. A pulse unrestricted use, distribution, and reproduction in any medium, provided the original work is properly cited. 
specifically tailored with attosecond precision such that it oscillates out-of-phase with the induced dipole leads to maximization of the interaction energy $\varepsilon(t)$. As a consequence, higher lying electronic target states (light-shaded area in Fig. 1), such as the $5^{1} \Sigma^{+}$g state, are energetically accessible and selectively excited. In-phase oscillation of laser and dipole result in a minimization of the interaction energy and lead to a selective excitation of lower lying electronic target states (darkshaded area in Fig. 1), such as the $4{ }^{1} \Sigma_{\mathrm{g}}^{+}$state. Quantummechanically, maximization (minimization) of $\varepsilon(t)$ is equivalent to the selective population of the upper (lower) light-induced potential of the resonant X-A-subsystem (bold light and thin dark curve in Fig. 1).

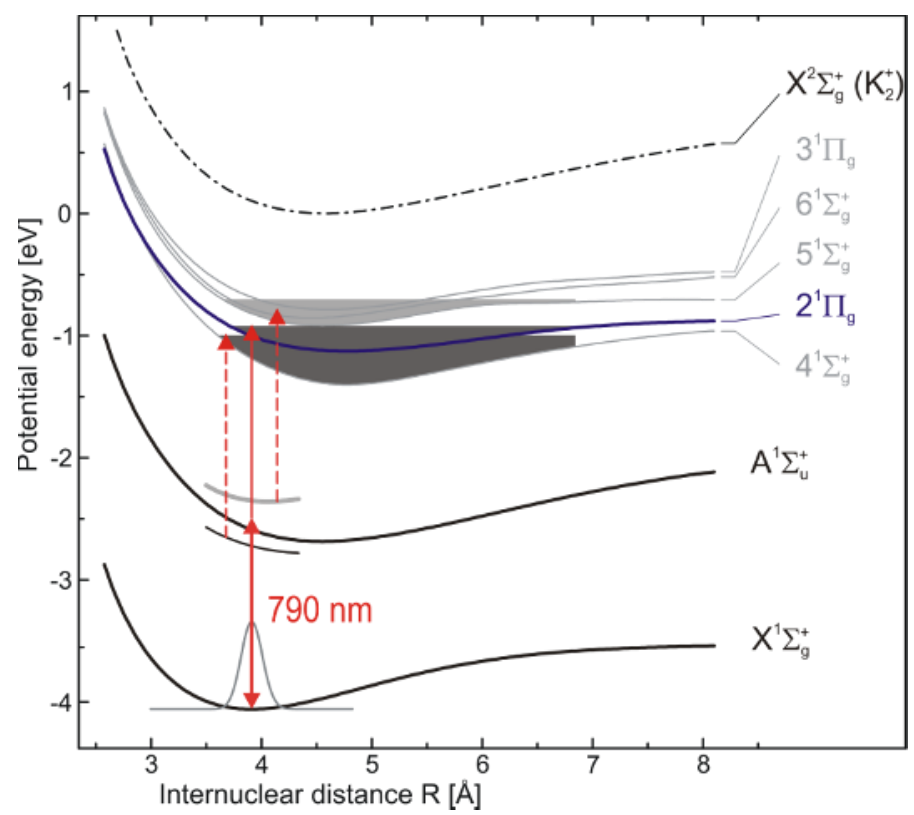

Fig. 1. Scheme for selective excitation of $\mathrm{K}_{2}$ with shaped $790 \mathrm{~nm} 25$ fs (Full-Width-HalfMaximum-duration) laserpulses (photon arrows). The pulses have a peak intensity of $\mathrm{I}=8.5 \cdot 10^{11} \mathrm{~W} / \mathrm{cm}^{2}$. In the weak-field limit the dimer is excited perturbatively from the groundstate $\mathrm{X}^{1} \Sigma_{\mathrm{g}}^{+}$via the resonant $\mathrm{A}^{1} \Sigma^{+}{ }_{\mathrm{u}}$ state to the $2^{1} \Pi_{\mathrm{g}}$ state. Resonant interaction with intense laserpulses couples the resonant states $\mathrm{X}$ and $\mathrm{A}$ efficiently and induces an electronic coherence, i.e. a molecular electronic wave packet is launched. The light-induced potentials (dark and light grey line) split up energetically and hence lower lying (dark shaded) and higher lying (light shaded) electronic target states become accessible.

\section{Efficient attosecond control}

The desired control pulse is generated by a home-built Fourier transform pulse shaper based on a Liquid Crystal Spatial Light Modulator (LC-SLM). A spectral phase mask of the form $\varphi(\omega)=A \sin \left[T\left(\omega-\omega_{0}\right)+\phi\right]$ is applied to the LC-SLM. This kind of spectral phase-modulation produces a temporal multi-pulse sequence and offers a large variety of different pulse shapes. After neutral excitation of the $\mathrm{K}_{2}$ a visible probe pulse ionizes the molecules and the ejected photoelectrons are detected. As a measure of the population in the target channels the population in the excited states is extracted by the integrated signal yields $S_{2 \Pi}$ and $S_{5 \Sigma}$. The contrast $C$ is determined according to $C=\left(S_{5 \Sigma}-S_{2 \Pi}\right) /\left(S_{5 \Sigma}+S_{2 \Pi}\right)$. $C$ ranges between -1 and 1 and indicates whether the $2^{1} \Pi_{\mathrm{g}}$ or the $5^{1} \Sigma_{\mathrm{g}}^{+}$state is efficiently populated. The contrast landscape that results as a 
function of the sine-frequency $T$ and the sine-phase $\phi$ is shown in Fig. 2. The landscape exhibits a pronounced maximum at $50 \mathrm{fs}$ and $\phi=\pi / 2$, corresponding to efficient population transfer to the upper target channel. By changing the sine-phase $\phi$ by $\pi$ an expanded valley can be found that is evidence for efficient population transfer to the lower target channel.

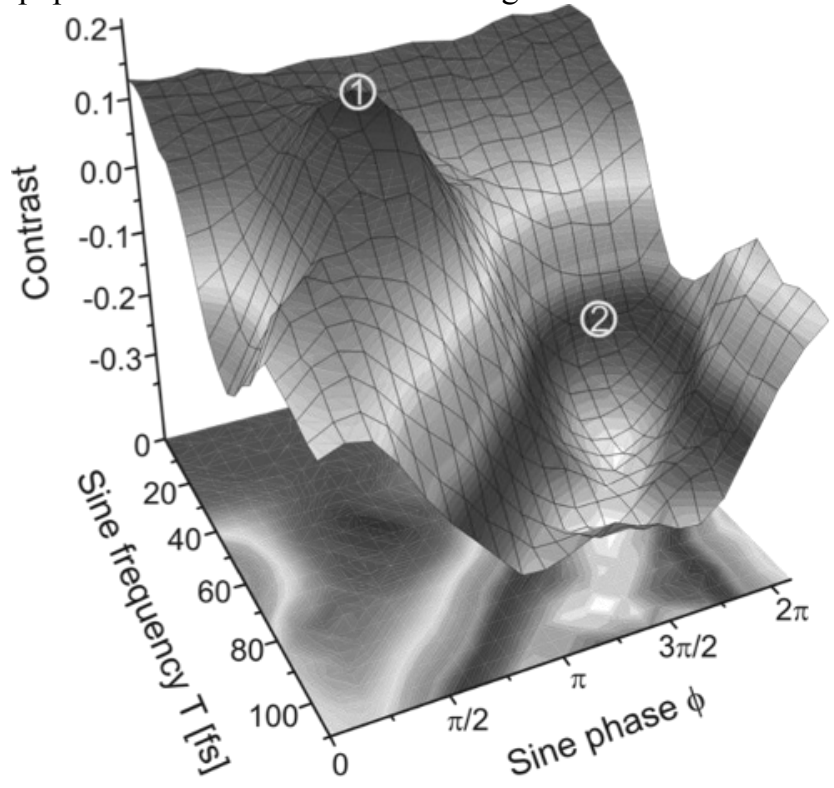

Fig. 2. Three-dimensional contrast landscape as it results from systematic variation of the sinefrequency $\mathrm{T}$ and the sine-phase $\Phi$. The sine-amplitude $\mathrm{A}$ was set to $0.8 \mathrm{rad}$. The landscape shows high contrast variations and particularly exhibits a distinct, isolated maximum around $\mathrm{T}=50 \mathrm{fs}$ and $\Phi=1.8 \mathrm{rad}$. At this point the modulated pulse excites the upper target channel with higher selectivity than the unmodulated pulse $(\mathrm{T}=0)$. Keeping $\mathrm{T}$ fixed a pure phase variation of $\Delta \Phi=\pi$ switches the population transfer in the molecule to the lower electronic target channel, as observed at $\Phi=4.8 \mathrm{rad}$.

In conclusion, this scenario allows to steer the system efficiently from its groundstate via a state of maximum electronic coherence towards a preselected electronic target channel which may lead to distinct subsequent nuclear dynamics. To control this electronic switching on an ultrafast timescale two ingredients must be combined. First, attosecond phase control is needed to adapt to the photoinduced dipole oscillations that are also subject to coupled electron-nuclear dynamics in a suitable way. Second, amplitude control is required to tune the interaction energy over a range of several 100 $\mathrm{meV}$ [4] in order to access the target states. Moreover the shown mechanism offers a wide spectrum of applications ranging from reaction control up to discrimination of nearly identical molecules. For the latter application ongoing theoretical work is in progress. In addition, first experiments on the controlled fragmentation of isopropyl alcohol show signatures of the presented control scheme also in larger molecules [5].

1. J. Köhler, M. Wollenhaupt, T. Bayer, C. Sarpe, T. Baumert, Opt. Expr. 19, 11638-11653 (2011)

2. M. Wollenhaupt, T. Baumert, J. Photochem. Photobiol. A 180, 248-255 (2006)

3. P. von den Hoff, M. Kowalewski, R. de Vivie-Riedle, Faraday Discuss. 153, 159-171 (2011).

4. M. Wollenhaupt, D. Liese, A. Präkelt, C. Sarpe-Tudoran, T. Baumert, Chem. Phys. Lett. 419, 184-190 (2006).

5. M. Wollenhaupt, T. Baumert, Faraday Discuss. 153, 9-26 (2011). 